\title{
On Directed Edge-Disjoint Spanning Trees in Product Networks, An Algorithmic Approach
}

\author{
A.R. Touzene* and K. Day \\ Department of Computer Science, College of Science, Sultan Qaboos University, P.O. Box 36, Postal Code 123, Al-Khodh, \\ Muscat, Sultanate of Oman.
}

Received 11 December 2013; accepted 15 September 2014

\begin{abstract}
In (Ku et al. 2003), the authors have proposed a construction of edge-disjoint spanning trees EDSTs in undirected product networks. Their construction method focuses more on showing the existence of a maximum number $\left(n_{1}+n_{2}-1\right)$ of EDSTs in product network of two graphs, where factor graphs have respectively $n_{1}$ and $n_{2}$ EDSTs. In this paper, we propose a new systematic and algorithmic approach to construct $\left(n_{1}+n_{2}\right)$ directed routed EDST in the product networks. The direction of an edge is added to support bidirectional links in interconnection networks. Our EDSTs can be used straightforward to develop efficient collective communication algorithms for both models store-and-forward and wormhole.
\end{abstract}

Keywords: Product networks, Directed edge-disjoint spanning trees, Interconnection networks.

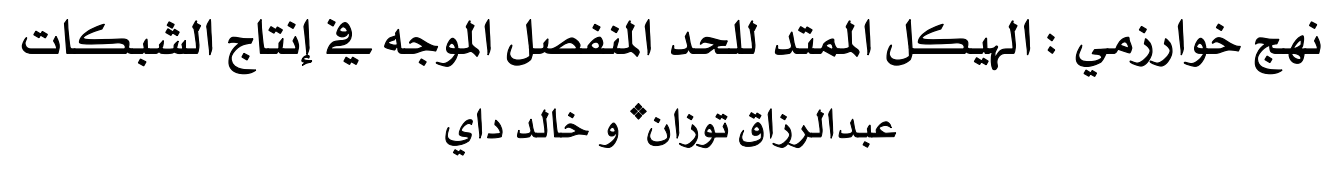

الملخص: اقترح المؤلفون بناء للهيكل الممتد للحدود المنفصلة EDSTs لإنتاج شبكات غير موجهة. طريقة البناء لديهم تركز أكثر على إظهار وجود الرقم الأقصى (n) EDST Eِ إنتاج الشبكات لرسمـين بيانيين حيث معامل الرسومات على الترتيب n1 و n2EDSTs. مِّ هذه المقالة نقدم مقترح لمنهج منظم ونظام حسابي جديد

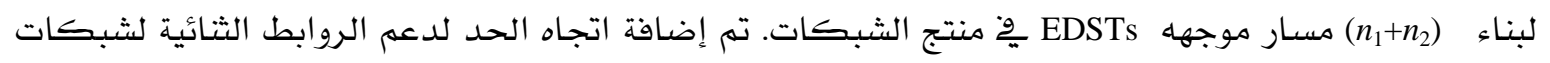
الربط. نظام EDSTs الخاص بنايمكن استخدامه مباشرة لتطوير خوارزميات الاتصالات الجماعية الفعالة لكل من نموذجي التخزين إلى الأمام و الثقب.

مفاتيح الكلمات: منتج الشبكات، الهيكل الممتد للحد المنفصل، الشبكات المتداخلة

*Corresponding author's e-mail: touzene@squ.edu.om 


\section{Introduction}

There has been increasing interest over the last two decades in product networks (Day, and Al-Ayyoub 1997; Ku et al. 2003; X and Yang 2007; Imrich et al. 2008; Klavar and Špacapan 2008; Jänicke et al. 2010; Hammack et al. 2011; Chen et al. 2011; Ma et al. 2011; Cheng et al. 2013; Erveš and Žerovnik 2013; Govorčin and Škrekovski 2014). The Cartesian product is a well-known graph operation. When applied to interconnection networks, the Cartesian product operation combines factor networks into a product network. Graph product is an important method to construct bigger graphs, and plays a key role in the design and analysis of networks. A number of spanning trees of a graph are edge-disjoint if no two trees contain the same edge. Edge-Disjoint spanning trees (EDSTs) have many practical applications including enhancing interconnection network fault-tolerance and developing efficient collective communication algorithms in distributed memory parallel computers (Fragopoulo and Akl 1996; Johnsson and Ho 1989; Touzene 2003). In ( $\mathrm{Ku}$ et al. 2003), the authorshave studied construction of maximum edge-disjoint spanning trees $\left(n_{1}+n_{2}-1\right)$ EDSTs in undirected product network of two graphs, where factor graphs have respectively $n_{1}$ and $n_{2}$ EDSTs. The presented construction is more about showing the existence of a maximum number of spanning trees. They did not provide a straight-forward algorithmic way for their construction. In this paper, we propose a new systematic and algorithmic approach to construct $\left(n_{1}+n_{2}\right)$ directed rooted edge-disjoint spanning tree in product networks. We assume that the factor graphs are connected graphs and have respectively $n_{1}$ and $n_{2}$ EDSTs. Directed rooted edge-disjoint spanning trees have been discussed for different graphs such as the ndimensional hypercube (Johnsson and Ho 1989), k-ary- $n$-cube (Touzene 2003), star graphs (Fragopoulo and Akl 1996), etc. We assume directed edges: if $a$ and $b$ are two nodes in the graph, the edge $(a, b)$ is different from the edge $(b, a)$. Directed edges support bidirectional links in interconnection networks. The advantage of our method is the direct use of our trees to develop collective communication procedures in product interconnection networks.

The remainder of this paper is organized as follows: In Section 2, notations and preliminaries are presented. In Section 3, the construction of edge-disjoint spanning trees in product networks is proposed. In Section 4, we conclude this paper.

\section{Notations and Preliminaries}

The Cartesian product $G=G_{1} \times G_{2}$ of two undirected graphs $G_{1}=\left(V_{1}, E_{1}\right)$ and $G_{2}=\left(V_{2}, E_{2}\right)$ is the undirected graph $G=(V, E)$, where $V$ and $E$ are given by: $V=\left\{\left\langle x_{1}, x_{2}\right\rangle \mid x_{1} \in V_{1}\right.$ and $\left.x_{2} \in V_{2}\right\}$, and for any $u=<x_{1}, x_{2}>$ and $\mathrm{v}=\left\langle y_{1}, y_{2}\right\rangle$ in $V,(u$, $v)$ is an edge in $E$ if, and only if, either $\left(x_{1}, y_{1}\right)$ is an edge in $E_{1}$ and $x_{2}=y_{2}$, or $\left(x_{2}, y_{2}\right)$ is an edge in $E_{2}$ and $x_{1}=y_{1}$. The edge $(u, v)$ is called a $G_{1}$-edge if $\left(x_{1}, y_{1}\right)$ is an edge in $G_{1}$, and it is called a $G_{2-}$ edge if $\left(x_{2}, y_{2}\right)$ is an edge in $E_{2} . x_{1}$ is called the $G_{1^{-}}$ component of $u$ and $x_{2}$ is called the $G_{2}$ component. In all what follows we consider directed edges in the sense that the edge $(u, v)$ is different from the edge $(v, u)$.

\section{Construction of EDSTs in a Product Network}

Consider two graphs $G_{1}=\left(V_{1}, E_{1}\right)$ and $G_{2}=$ $\left(V_{1}, E_{1}\right)$ having the following properties: the graph $G_{1}$ contains $n_{1}$ EDST all rooted at $x$ denoted: $X_{1}(x), X_{2}(x), \ldots, X_{n 1}(x)$. Each $X_{i}(x)$ tree is assumed to be formed of an edge $\left(x, x_{i}\right)$, where $x_{i}$ is the $i^{\text {th }}$ neighbor of $x$, and a sub-tree denoted $X_{i}(x) / x$ rooted at $x_{i}$ that spans all the $G_{1}$ nodes other than $x$ (Fig. 1.a). The graph $G_{2}$ contains $n_{2}$ EDST all rooted at $y$ denoted: $Y_{1}(y), Y_{2}(y), \ldots$, $Y_{n 2}(y)$, Each $Y_{j}(y)$ tree is assumed to be formed of an edge $\left(y, y_{j}\right)$, where $y_{j}$ is the $j^{\text {th }}$ neighbor of $y$, and a sub-tree denoted $Y_{j}(y) / y$ rooted at $y_{j}$ that spans all the $G_{2}$ nodes other than $y$ (figure 1.b). In Fig. $1(a, b)$ straight lines correspond to $G_{1}$ edges and dashed lines correspond to $G_{2}$-edges. 


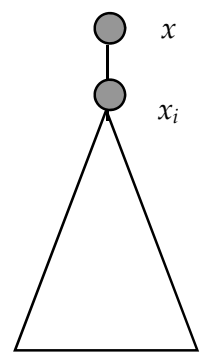

\section{$X_{i}(x / x) y_{i}(y / y)$}

Figure 1.a. $i$ th $\operatorname{EDSTX}_{i}(x)$ rooted at $x$ in $G_{1}$ and its $X_{i}(x)$ sub-tree.

In what follows, we fix a specific node $<x_{0}$, $y_{0}>$ in $G$ as a desired root for the EDST to be constructed. We denote by $\left\langle x_{i}, y_{0}\right\rangle, i=1, \ldots, n_{1}$, the $n_{1}$ neighbors of $\left\langle x_{0}, y_{0}>\right.$ in $G$ reached from $\left.<x_{0}, y_{0}\right\rangle$ via $G_{1}$-edges, and by $\left\langle x_{0}, y_{j}\right\rangle, j=1, \ldots, n_{2}$, the $n_{2}$ neighbors of $<x_{0}, y_{0}>$ reached from $<x_{0}$, $y_{0}>$ via $G_{2}$-edges. For a given node $x$ in $G_{1}$ and a given tree $Y$ in $G_{2}$, we denote by $\langle x, Y>$ the tree in $G_{1} \times G_{2}$ obtained by fixing the $G_{1}$-component to $x$ and following the edges of tree $Y$ in $G_{2}$. Similarly, $\left\langle X, y>\right.$ denotes the tree in $G_{1} \times G_{2}$ obtained by following the edges of a tree $X$ in $G_{1}$ while the $G_{2}$-component is fixed to node $y$.

\subsection{The $S T_{1}$ and $S T_{2} E D S T$ for $G$}

We present a construction algorithm of $n_{1}+n_{2}-2$ EDST (without using non-tree edges (Ku et al. 2003) for the product graph G: $n_{1}-1$ EDST for $G$ denoted $\mathrm{ST}_{1}(i), i=2 . . n_{1}$ and $n_{2}$-1EDST for $G$ denoted $\mathrm{ST}_{2}(j), j=2 . . n_{2}$.

\subsection{Construction of $S T_{1}(i)$, for any $i 2 \leq i \leq n_{1}$}

1. Connect $\left\langle x_{0}, y_{0}\right\rangle$ to its neighbor $\left\langle x_{i}, y_{0}\right\rangle$ (see edge labeled 1 in Fig. 2(a)).

2. Attach to $\left\langle x_{i}, y_{0}\right\rangle$ the sub-tree $\left\langle X_{i}\left(x_{0}\right) / x_{0}\right.$, $y_{0}>$ (see sub-tree labeled 2 in Fig. 2(a)).

3. Connect $\left\langle x_{i}, y_{0}\right\rangle$ to its neighbor $\left\langle x_{i}, y_{1}\right\rangle$ (see edge labeled 3 in Fig. 2(a)).

4. To $\left\langle x_{i}, y_{1}\right\rangle$ attach the sub-tree $<x_{i}$, $Y_{1}\left(y_{0}\right) / y_{0}>$ (see sub-tree labeled 4 in Fig. 2(a)).

5. To each node $\left\langle x_{i}, y>\right.$ in the sub-tree $<x_{i}$, $Y_{1}\left(y_{0}\right) / y_{0}>$ (including its root $<x_{i}, y_{1}>$ ) attach the tree $\left\langle X_{i}\left(x_{0}\right) / x_{0}, y\right\rangle$ (see sub-tree labeled 5 in Fig. 2(a)).

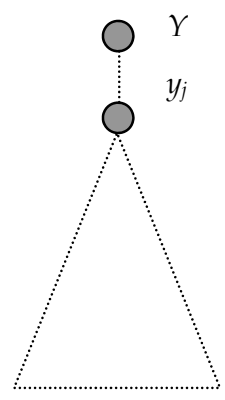

Figure 1.b. ith EDST $Y_{i}(y)$ at y in $G_{2}$ and its $Y_{j}(y) / y$ sub-tree.

6. Connect each node $\left\langle x_{i}, y>\right.$ in the subtree $<x_{i}, Y_{1}\left(y_{0}\right) / y_{0}>$ (including its root $<x_{i}$, $y_{1}>$ ) to its neighbor $\left\langle x_{0}, y_{1}>\right.$ (see edge labeled 6 in Fig. 2(a)).

3.3 Construction of the tree $S T_{2}(j), j=2, . . n_{2}$

1. Connect $\left\langle x_{0}, y_{0}\right\rangle$ to its neighbor $\left\langle x_{0}, y_{j}\right\rangle$ (see edge labeled 1 in Fig. 2(b)).

2. Attach to $\left\langle x_{0}, y_{j}\right\rangle$ the sub-tree $<x_{0}, Y_{j}\left(y_{0}\right) / y_{0}>$ (see sub-tree labeled 2 in Fig. 2(b)).

3. Connect $\left\langle x_{0}, y_{j}\right\rangle$ to its neighbor $\left\langle x_{1}, y_{j}\right\rangle$ (see edge labeled 3 in Fig. 2(b)).

4. To $\left\langle x_{1}, y_{\mathrm{j}}>\right.$ attach the sub-tree $\left.<X_{1}\left(x_{0}\right) / x_{0}, y_{\mathrm{j}}\right\rangle$ (see labeled 4 in Fig. $2(b))$.

5. To each node $<x, y_{\mathrm{j}}>$ in the sub-tree $<X_{1}\left(x_{0}\right) / x_{0}, y_{\mathrm{j}}>$ (including its root $<x_{1}$, $y_{j}>$ ) attach the tree $\left\langle x, Y_{j}\left(y_{0}\right) / y_{0}\right\rangle$ (see sub-tree labeled 5 in Fig. 2(b)).

6. Connect each node $<x, y_{\mathrm{j}}>$ in the subtree $<X_{1}\left(x_{0}\right) / x_{0}, y_{\mathrm{j}}>$ (including its root $\left\langle x_{1}, y_{j}\right\rangle$ ) to its neighbor $\left\langle x_{1}, y_{0}\right\rangle$ (see edge labeled 6 in figure 2(b)).In figure 2(a, b), straight lines are $G_{1}$-edges and dashed lines are to $G_{2}$-edges.

Theorem 1: The set $\left\{\mathrm{ST}_{1}(i), 2 \leq i \leq n_{2}\right\} \cup\left\{\mathrm{ST}_{2}(j), 2 \leq\right.$ $\left.j \leq n_{2}\right\}$ is a family of $\left(n_{1}+n_{2}-2\right)$ edge-disjoint panning trees in $G=G_{1} \times G_{2}$.

Proof: We show that all the nodes $\langle x, y\rangle$ of the product graph are reached in the $\left(n_{1}+n_{2}-2\right)$ edgedisjoint spanning tree using different edges. 


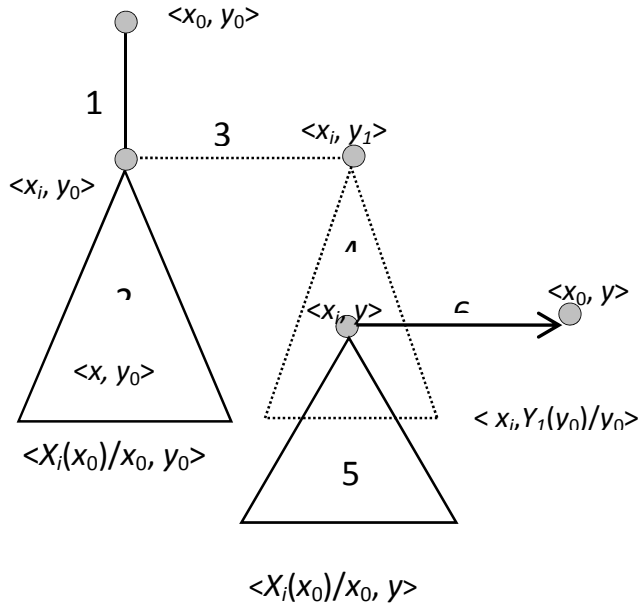

(a) $\mathrm{ST}_{1}(i), i=2 . . n_{1}$

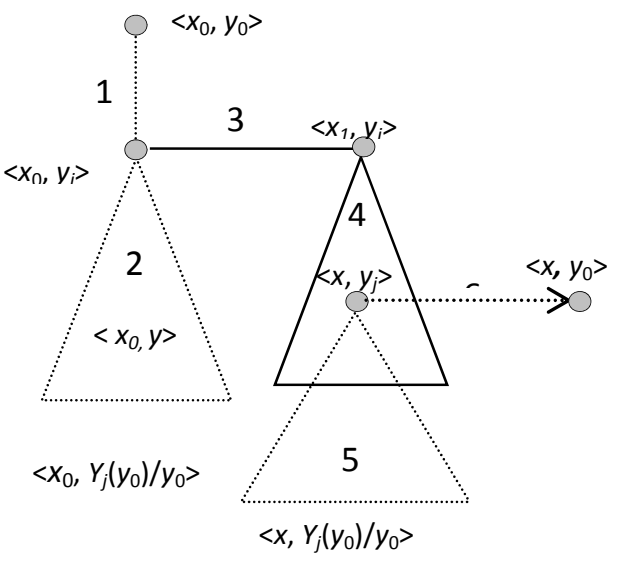

(b) $\mathrm{ST}_{2}(j), j=2 . . n_{2}$

Figure 2. Construction of spanning trees $\mathrm{ST}_{1}(i)$ and $\mathrm{ST}_{2}(j)$.

- Case 1: nodes $\left\langle x_{0}, y\right\rangle$ are reached by different $G_{1}$-edges $\left.\left(\left\langle x_{i}, y\right\rangle,<x_{0}, y\right\rangle\right), i=2, \ldots$, $n_{1}$, in the different trees $\mathrm{ST}_{1}(i)$ (edges labeled 6 in figure 2(a)). In trees $\mathrm{ST}_{2}(j), j=2$, $\ldots, n_{2}$, these nodes are covered by $G_{2}$-edges of the sub-trees $<x_{0}, \quad Y_{j}\left(y_{0}\right) / y_{0}>$ (edges labeled 2 in Fig. 2(b)).

- Case 2: nodes $\left\langle x, y_{0}\right\rangle$, similar proof as in case 1 (symmetrical).

- $\quad$ Case 3: nodes $\left\langle x_{i}, y\right\rangle, i=2, \ldots, n_{1}$ are covered in four different ways:

1. In sub-trees $<x_{i}, Y_{1}\left(y_{0}\right) / y_{0}>, i=2, \ldots, n_{1}$ of trees $\mathrm{ST}_{1}(i)$ using $Y_{1}$ tree edges (labeled 4 Fig. 2(a)).

2. In sub-tree $<x, Y_{j}\left(y_{0}\right) / y_{0}>, j=2, \ldots, n_{2}$ of the trees $\mathrm{ST}_{2}(j)$. These nodes are covered using $Y_{j}$ trees edges $(j>1)$, (labeled 5 in Fig. 2(b)).

3. In sub-trees $\left\langle X_{i}\left(x_{0}\right) / x_{0}, y\right\rangle, i=2, \ldots, n_{1}$ of trees $\mathrm{ST}_{1}(j)$ using $X_{i}$ tree edges (labeled 5 in Fig. 2(a)) .

4. In sub-tree $<X_{1}\left(x_{0}\right) / x_{0}, y_{j}>j=2, \ldots, n_{2}$ of the trees $\mathrm{ST}_{2}(j)$ using $X_{1}$ treeedges(labeled 4 in Fig. 2(b)).

- Case 4: nodes $\left\langle x, y_{j}\right\rangle$, similar proof as in case 3 (symmetrical).

- $\quad$ Case 5: nodes $\langle x, y\rangle, x \neq x_{i}, y \neq y_{j}$ are covered using different $G_{1}$-edges in sub-trees $<X_{i}\left(x_{0}\right) / x_{0}, y>, i=2, \ldots, n_{1}$ of trees $\mathrm{ST}_{1}(i)$ (sub- tree labeled 5 in Fig. 2(a)). These nodes are covered using $G_{2}$-edges in the sub-trees $<x$, $Y_{\mathrm{j}}\left(y_{0}\right) / y_{0}>, j=2, \ldots, n_{2}$ in the trees $\mathrm{ST}_{2}(j)$ (labeled 5 in Fig. 2(b)).

\subsection{The Special $T_{1}$ and $T_{2}$ EDSTs for $G$}

We present a construction algorithm for the directed EDSTs in the product graph $G$ denoted $\mathrm{T}_{1}$ and $\mathrm{T}_{2}$.

\subsection{Construction of $T_{1}$}

1. Connect $<x_{0}, y_{0}>$ to its neighbor $<x_{1}$, $y_{0}>$ (see edge labeled 1 in Fig. 3(a)).

2. Attach to $\left\langle x_{1}, y_{0}\right\rangle$ the sub-tree $<X_{1}\left(x_{0}\right) / x_{0}, y_{0}>$ (see sub-tree labeled 2 in Fig. 3(a)).

3. Connect $\left\langle x_{1}, y_{0}>\right.$ to its neighbor $\left\langle x_{1}, y_{1}\right\rangle$ (see edge labeled 3 in Fig. 3(a)).

4. To $<x_{1}, y_{1}>$ attach the sub-tree $<x_{1}$, $Y_{1}\left(y_{0}\right) / y_{0}>$ (see sub-tree labeled 4 in Fig. 3(a)).

5. To each node $\left\langle x_{1}, y / y_{\mathrm{j}}\right\rangle, j=1, \ldots, n_{2}$ in the sub-tree $<x_{1}, Y_{1}\left(y_{0}\right) / y_{0}>$ (including its root $\left\langle x_{1}, y_{1}>\right.$ ) attach the tree $\left\langle X_{1}\left(x_{0}\right) / x_{0}\right.$, $y>$ (see sub-tree labeled 5 in Fig. 3(a)).

6. Connect each node $\left\langle x_{1}, y\right\rangle$ in the subtree $<x_{1}, \quad Y_{1}\left(y_{0}\right) / y_{0}>$ (including its root $<x_{1}, y_{1}>$ ) to its neighbor $<x_{0}, y_{1}>$ (see edge labeled 6 in Fig. 3(a)). 


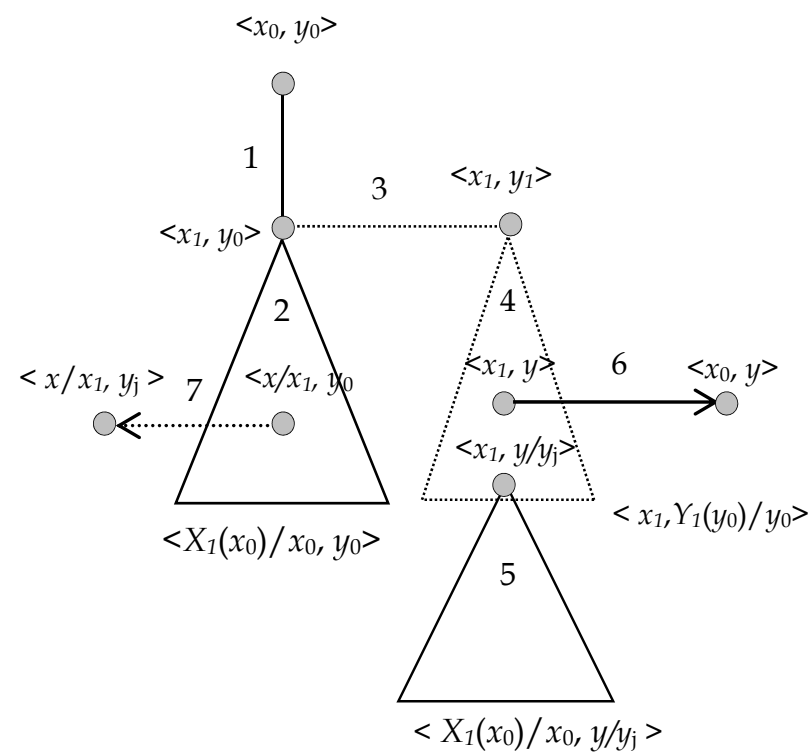

(a) $T_{1}$

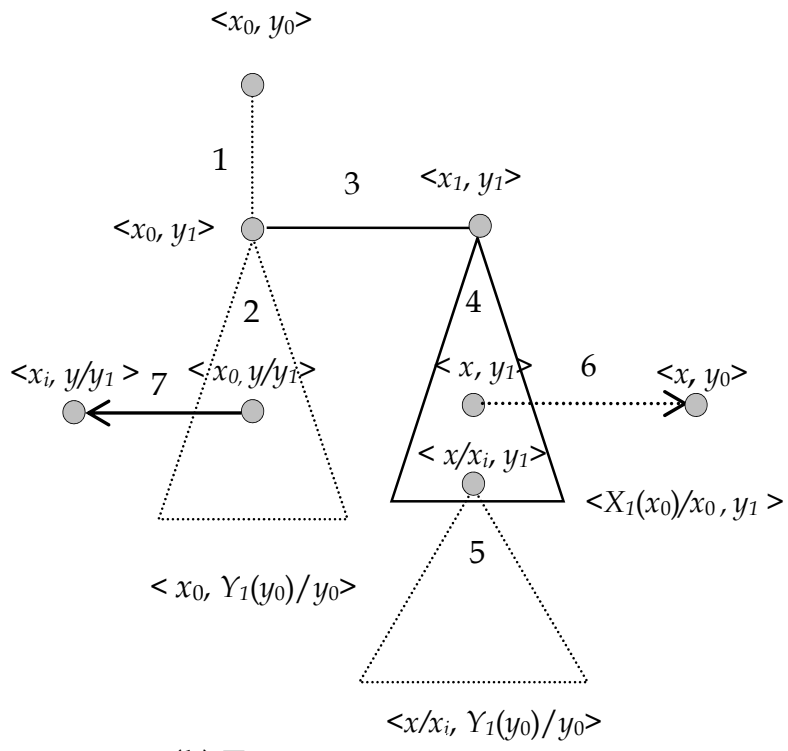

(b) $T_{2}$

Figure 3. Construction of spanning trees $\mathrm{T}_{1}$ and $\mathrm{T}_{2}$.

7. Connect each node $\left\langle x_{1}, y\right\rangle$ in the subtree $<x_{1}, \quad Y_{1}\left(y_{0}\right) / y_{0}>$ (including its root $\left.<x_{1}, y_{1}>\right)$ to its neighbor $<x_{0}, y_{1}>$ (see edge labeled 6 in Fig. 3(a)).

8. Connect each node $\left\langle x / x_{1}, y_{0}\right\rangle$ in the subtree $<X_{1}\left(x_{0}\right) / x_{0}, \quad y_{0}>$ to the node $<x / x_{1}$, $y_{j}>$ (see label 7 in Fig. 3(a)).

\subsection{Construction of the Tree $T_{2}$}

1. Connect $\left\langle x_{0}, y_{0}>\right.$ to its neighbor $\left\langle x_{0}, y_{1}\right\rangle$ (see edge labeled 1 in Fig. 3(b)).

2. Attach to $\left\langle x_{0}, y_{1}\right\rangle$ the sub-tree $<x_{0}, Y_{1}\left(y_{0}\right) / y_{0}>$ (see sub-tree labeled 2 in Fig. 3(b)).

3. Connect $\left\langle x_{0}, y_{1}\right\rangle$ to its neighbor $\left\langle x_{1}, y_{1}\right\rangle$ (see edge labeled 3 in Fig. 3(b)).

4. To $\left\langle x_{1}, y_{1}>\right.$ attach the sub-tree $<X_{1}\left(x_{0}\right) / x_{0}, y_{1}>$ (see labeled 4 in Fig. 3(b)).

5. To each node $<x / x_{\mathrm{i}}, y_{1}>, i=1, \ldots, n_{1}$ in the sub-tree $\left\langle X_{1}\left(x_{0}\right) / x_{0}, y_{1}>\right.$ (including its root $\left.<x_{1}, y_{1}>\right)$ attach the tree $<x$, $Y_{1}\left(y_{0}\right) / y_{0}>$ (see sub-tree labeled 5 in Fig. $3(b))$.
6. Connect each node $<x, y_{1}>$ in the subtree $<X_{1}\left(x_{0}\right) / x_{0}, y_{1}>$ (including its root $\left.\left\langle x_{1}, y_{1}\right\rangle\right)$ to its neighbor $\left\langle x_{1}, y_{0}\right\rangle$.

7. Connect each node $\left\langle x_{0}, y / y_{1}\right\rangle$ in the subtree $<x_{0}, Y_{1}\left(y_{0}\right) / y_{0}>$ to the node $<x_{i}$, $y / y_{1}>$ (see label 7 in Fig. $3(b)$ ).

Note that in $T_{1}$ the edges $\left(\left\langle x, y_{0}\right\rangle,\left\langle x, y_{j}\right\rangle\right)$ are used but in $T_{2}(j), 2 \leq j \leq n_{2}$, the opposite direction edges $\left.\left(<x, y_{j}>\right),<x, y_{0}>\right)$ are use. Similarly, in $\mathrm{T}_{2}$ the edges $\left(\left\langle x_{0}, y\right\rangle,\left\langle x_{i}, y\right\rangle\right)$ are used but in $\mathrm{T}_{1}(i), 2 \leq i \leq n_{1}$, the opposite direction edges $\left(\left\langle x_{i}, y\right\rangle,\left\langle x_{0}, y\right\rangle\right)$ are used. It is easy to see that using a similar proof as in Theorem 1, the trees $\mathrm{T}_{1}, \mathrm{~T}_{2}, \mathrm{ST}_{1}(i), 2 \leq i \leq n_{2}$ and $\mathrm{T}_{2}(j), 2 \leq j \leq n_{2}$ is a family of $\left(n_{1}+n_{2}\right)$ directed rooted edge-disjoint spanning trees in $G=$ $G_{1} \times G_{2}$.

To illustrate our construction algorithm, we give a complete example of product of two interconnection networks the 3-cube (3 directed rooted EDTS's (Johnsson and Ho 1989)) and a ring with three nodes $(\mathrm{a}, \mathrm{b}$ and $\mathrm{c})$ (2 directed rooted EDST's). Dark circles represents the root node of the trees and the numbers on the edges 

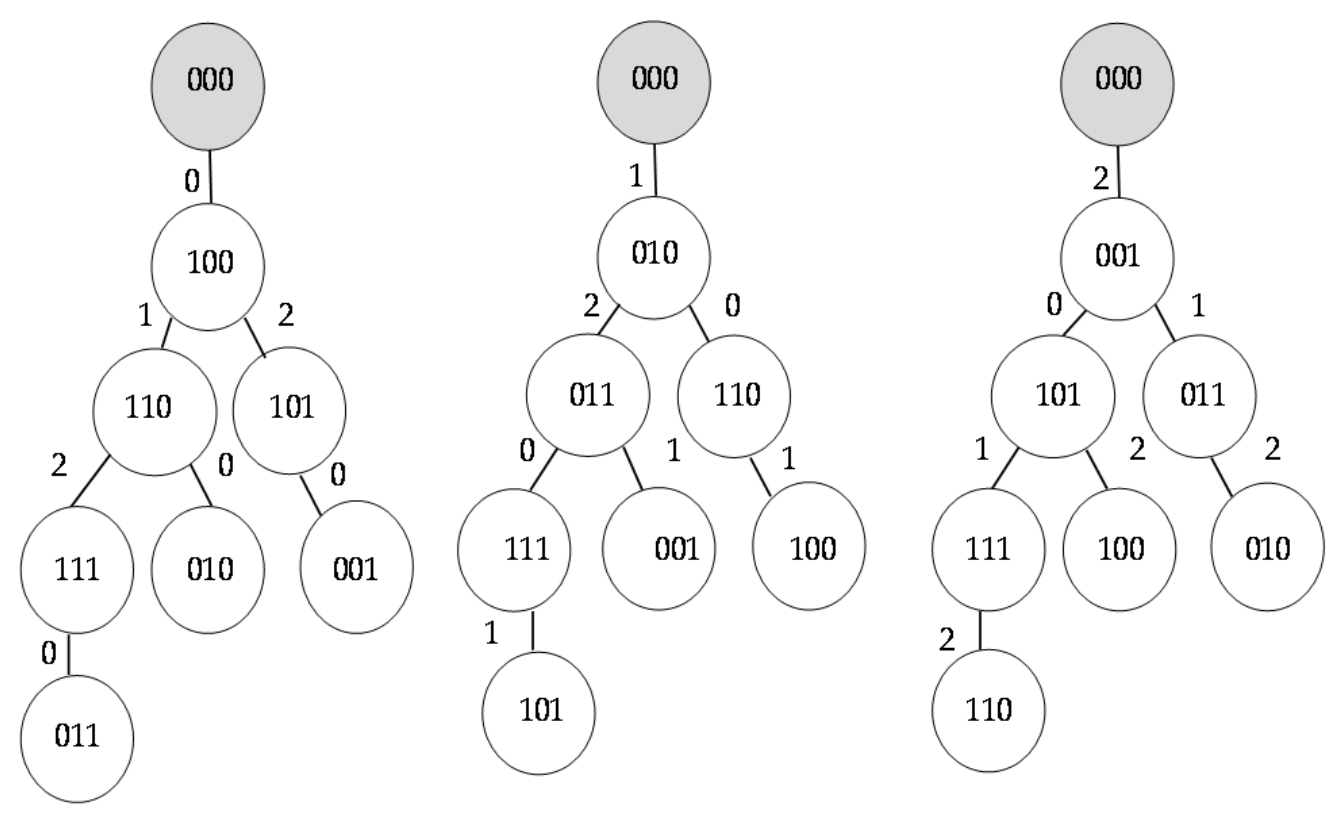

$X_{1}$

$\mathrm{X}_{2}$

$X_{3}$
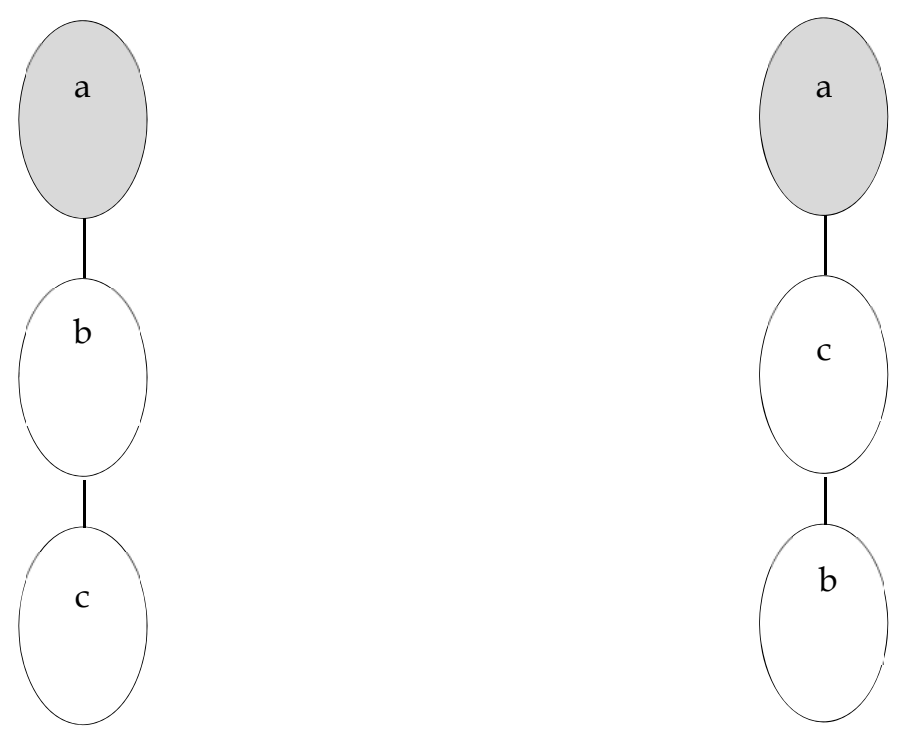

$\mathrm{Y}_{1}$ $\mathrm{Y}_{2}$

Figure 4. Three EDSTs of the 3-cube and two EDSTs of the ring (3 nodes). 


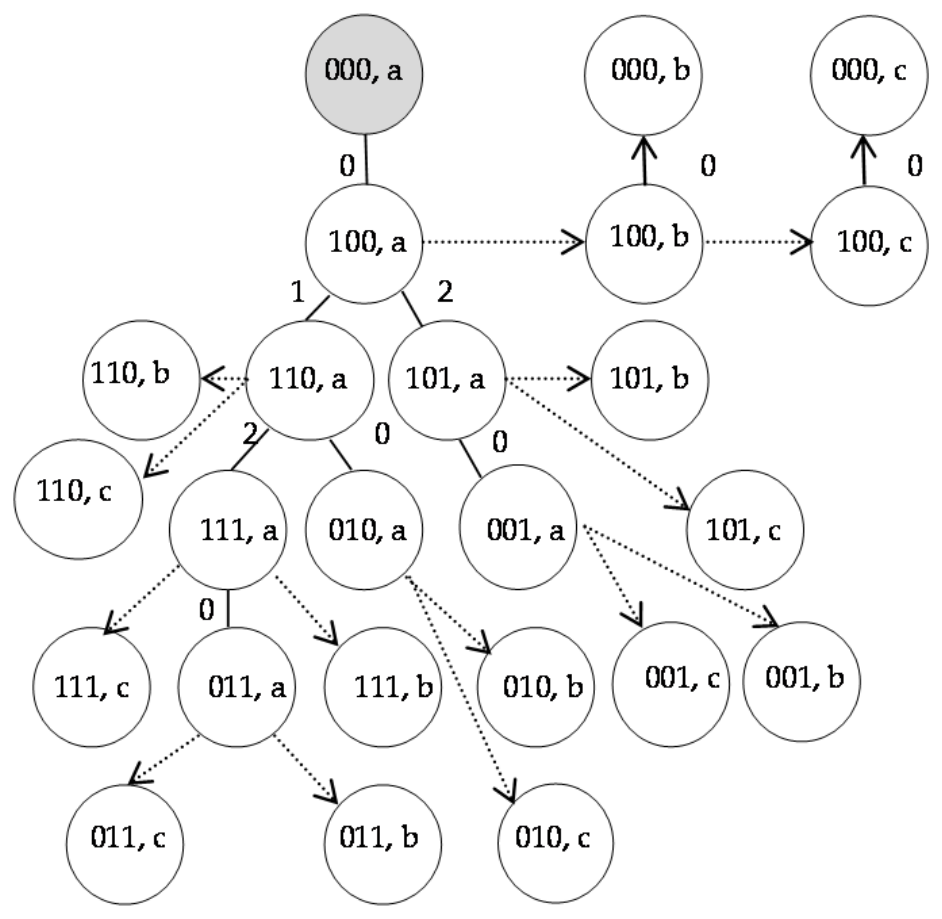

Figure 5 (a).Spanning $\operatorname{Tree~} \mathrm{ST}_{1}(1)$.

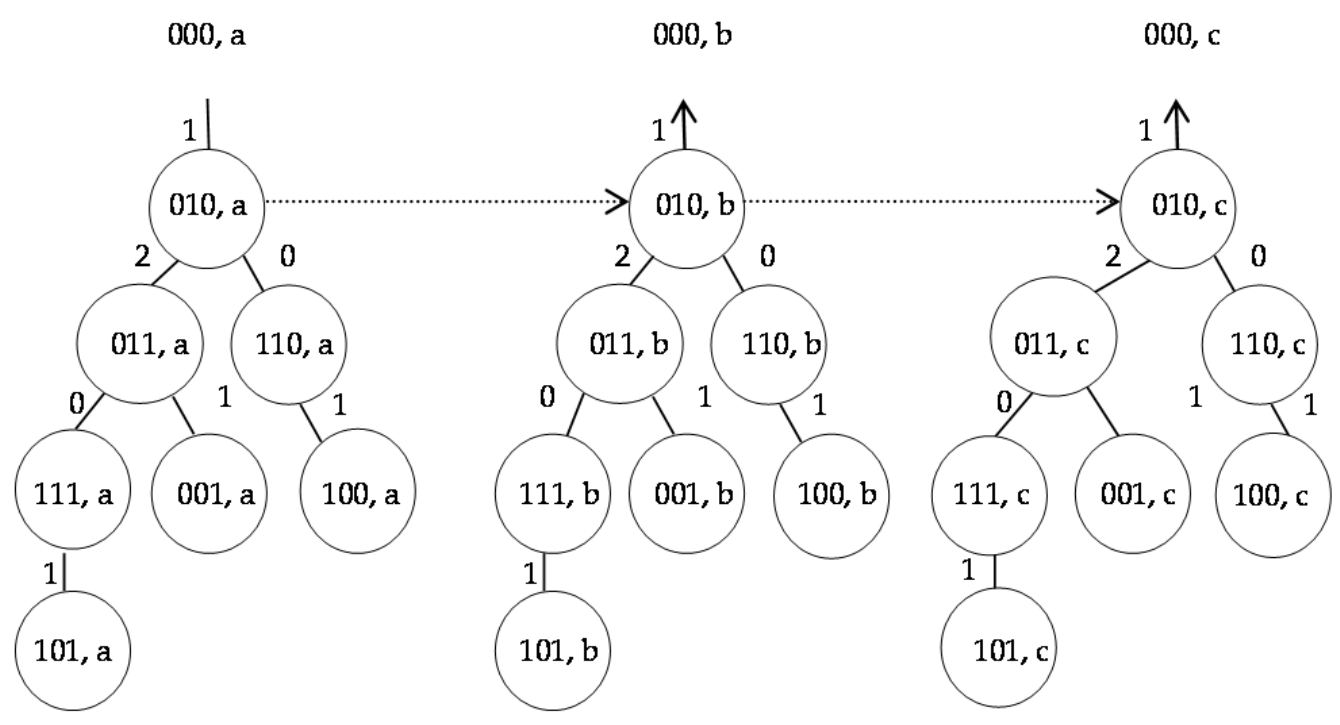

Figure 5 (b). Spanning Tree $\mathrm{ST}_{1}(2)$. 


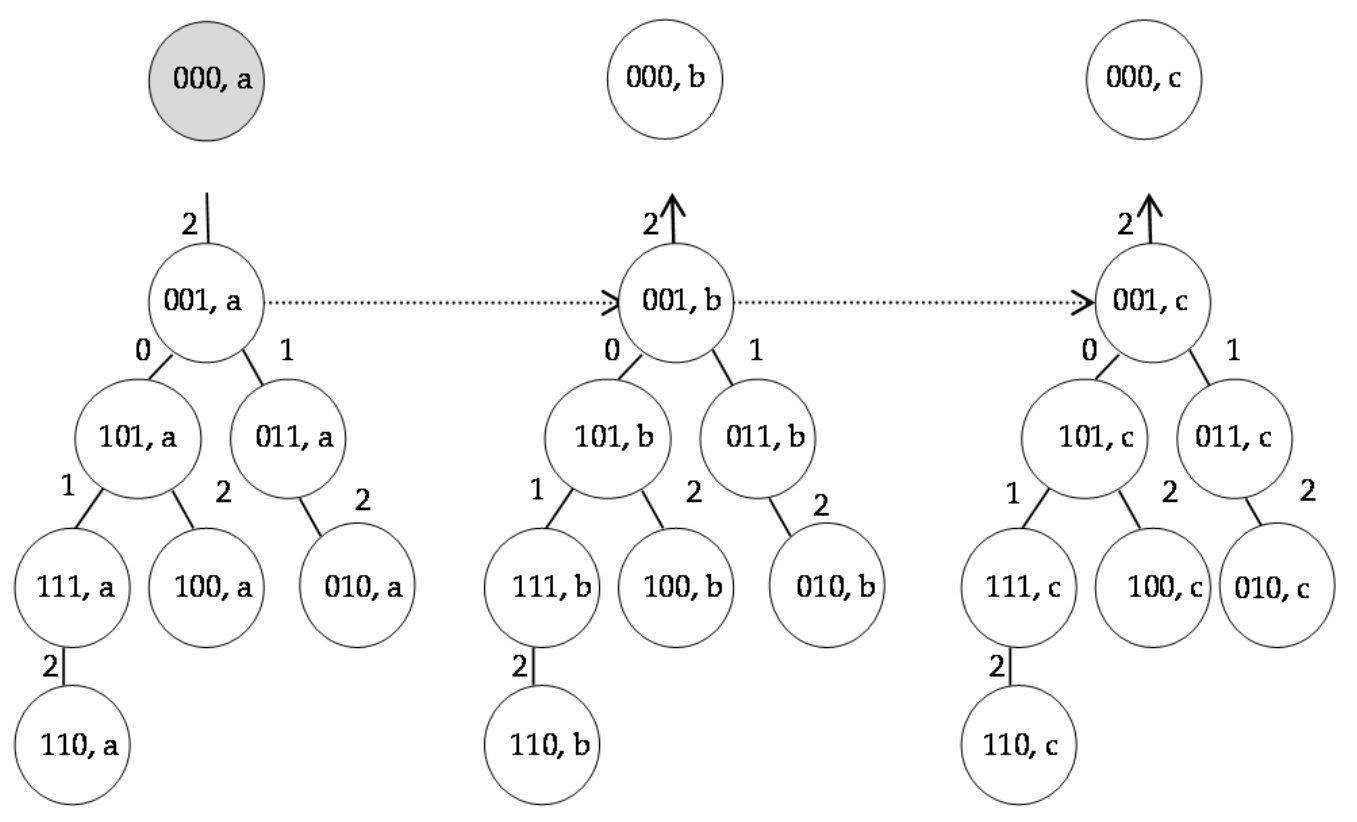

Figure 5 (c). Spanning Tree $\mathrm{ST}_{1}(3)$.

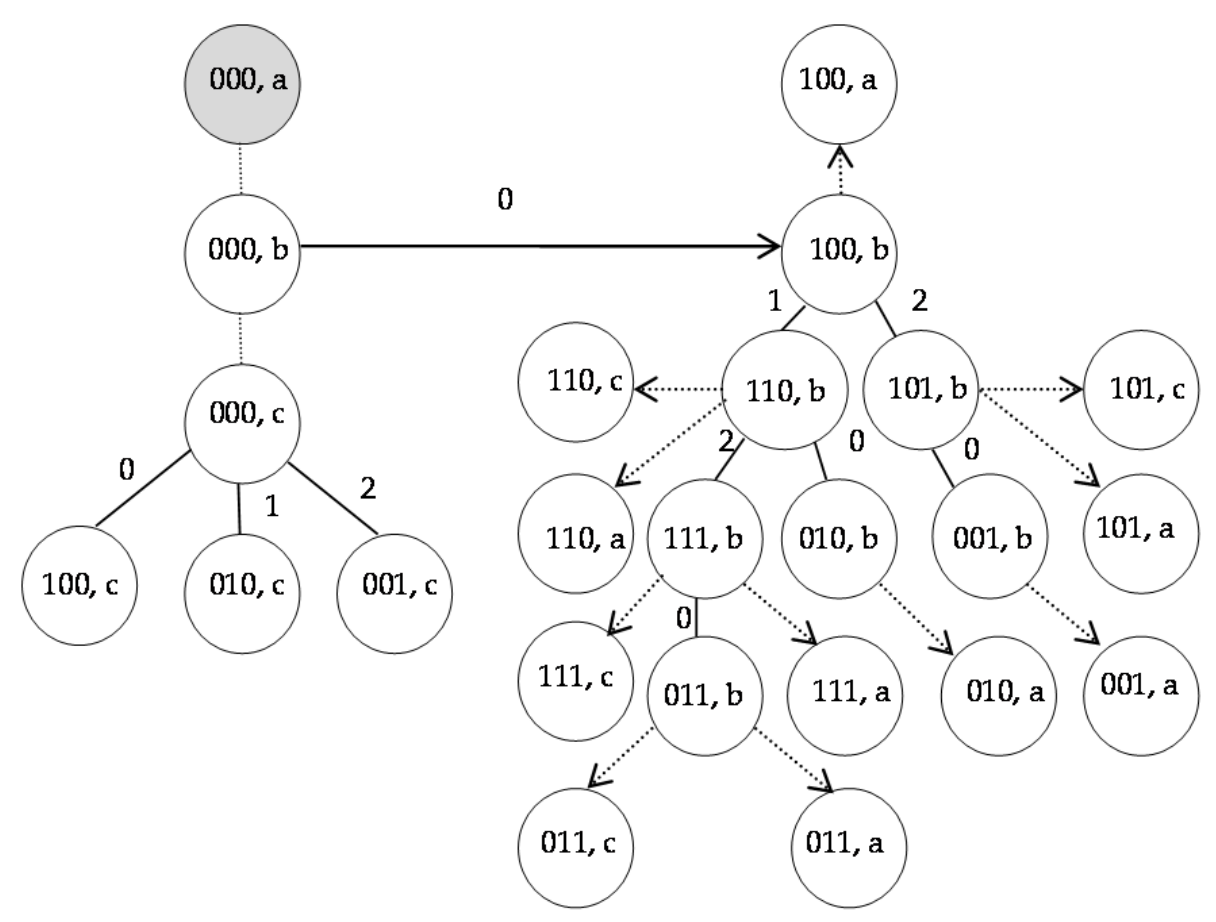

Figure 5 (d). Spanning Tree $\mathrm{ST}_{2}(1)$. 


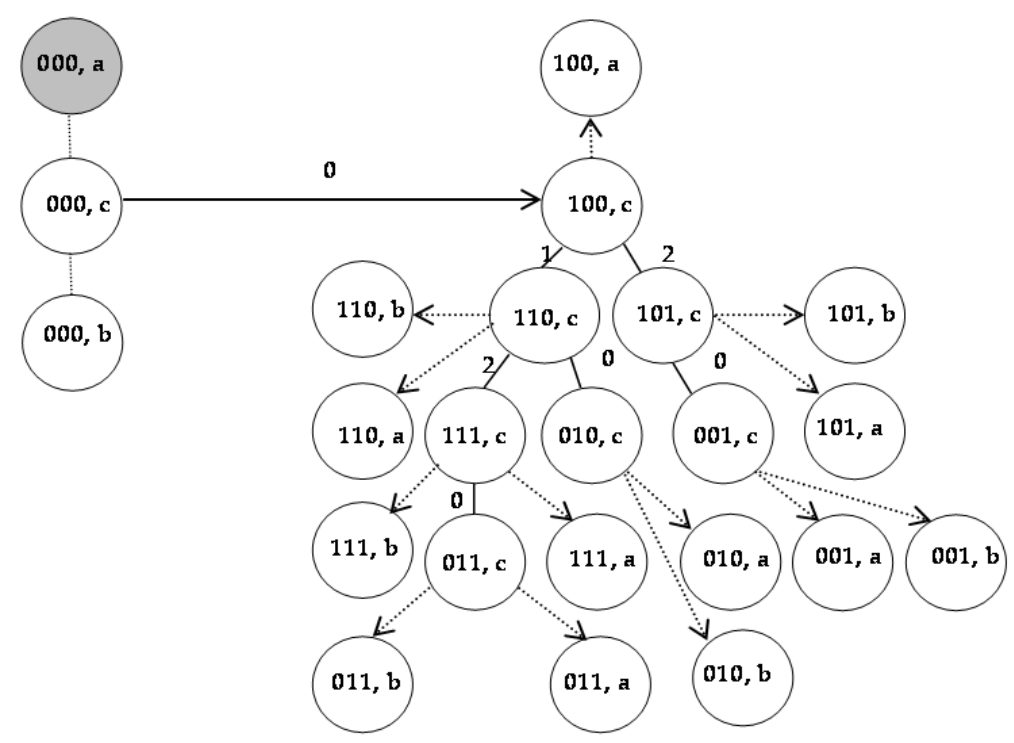

Figure 5 (e). Spanning Tree $\mathrm{ST}_{2}(2)$.

represent the dimension number relative to the 3-cube, see Figs. 4 and 5. The trees are directed from the root nodes to leave nodes.

\section{Conclusions}

In this paper, we presented a new systematic and algorithmic approach to construct $n_{1}+n_{2}$ (without using non-tree edges) directed rooted edges-disjoint spanning trees for product networks. The previous work on undirected EDSTs of the product networks (Ku et al. 2003) focuses more on the existence of $n_{1}+n_{2}-1$ but did not provide an explicit algorithmic way for their construction. Our $n_{1}+n_{2}$ EDSTs can be used straight-forward to develop efficient collective communication algorithms for both models store-and-forward and wormhole using bidirectional links.

\section{References}

Chen M, GuoX, Zhai S (2011), The optimal strong radius and optimal strong diameter of the Cartesian product graphs. Applied Mathematics Letters 24(5):657-660.
Cheng CW, Lee CW, Hsieh SY (2013), Conditional edge-fault hamiltonicity of cartesian product graphs. IEEE Transactions on Parallel and Distributed Systems 24 (10):1951-1960.

Day K, Al-Ayyoub AE (1997) , The Cross Product of Interconnection Networks. IEEE Trans. Parallel and Distributed Systems 8(2):109-118.

Erveš R, Žerovnik J (2013), Mixed fault diameter of Cartesian graph bundles. Discrete Applied Mathematics 161 (12):1726-1733.

Fragopoulo P, Akl SG (1996), Edge-disjoint spanning trees on the star network with application to fauttolerance. IEEE Trans. Computers 45(2):174-185.

Govorčin J, Škrekovski R (2014) , On the connectivity of Cartesian product of graphs. ArsMathematicaContemporanea 7(2):293297.

Hammack R, Imrich W, Klavžar S (2011), Handbook of Product Graphs,Discrete Mathematics and Its Applications, Second ed. CRC Press Boca Raton.

Imrich W, Klavžar S, Rall DF (2008), Topics in Graph Theory: Graphs and their Cartesian Product, AK Peters, Ltd., Wellesley, MA. 
Jänicke S, Heine C, Hellmuth M, Stadler PF, Scheuermann G (2010), Visualization of graph products. IEEE Transactions on Visualization and Computer Graphics 16(6):1082-1089.

Johnsson SL, Ho CT (1989) , Optimal broadcasting and personalized communication in hypercubes. IEEE Trans. Computers 38(9):1249-1268.

Klavar S, Špacapan S (2008), On the edgeconnectivity of cartesian product graphs. Asian-European Journal of Mathematics 1(1):93-98.

Ku S, Wang B , Hung T (2003), Constructing edge-disjoint spanning trees in product networks. IEEE Trans. Parallel and Distributed Systems 14(3):213-221.
Ma M,Xu JM, Zhu Q (2011),The menger number of the cartesian product of graphs. Applied Mathematics Letters 24(5):627-629.

Touzene A (2004), Optimal all-port collective communication algorithms for the k-ary $\mathrm{n}$ cube interconnection networks. Journal of Systems Architecture 50:221-231.

Xu JM, Yang C(2007), Fault diameter of product graphs. Information Processing Letters 102(6):226-228. 